\title{
More identities on Fibonacci and Lucas hybrid numbers
}

\author{
Nazmiye Yilmaz \\ Department of Mathematics, University of Karamanoğlu Mehmetbey \\ Kamil Özdă̆ Science Faculty, Turkey \\ e-mail: yilmaznzmy@gmail.com
}

Received: 17 August 2020

Revised: 8 April 2021

Accepted: 3 May 2021

\begin{abstract}
We give several identities about Fibonacci and Lucas hybrid numbers. We introduce the Fibonacci and Lucas hybrid numbers with negative subscripts. We obtain different Cassini identities for the conjugate of the Fibonacci and Lucas hybrid numbers by two different determinant definitions of a hybrid square matrix (whose entries are hybrid numbers).
\end{abstract}

Keywords: Binomial, Conjugate, Fibonacci hybrid number, Lucas hybrid number.

2020 Mathematics Subject Classification: 11B39, 97F50.

\section{Introduction}

Complex, hyperbolic and dual numbers are well known two dimensional number systems. Especially in the last century, a lot of researchers deal with the geometric and physical applications of these numbers. A hybrid number can be viewed as a generalization of the complex, hyperbolic and dual number. The set of hybrid numbers in [9], denoted by $\mathbb{K}$, is defined as

$$
\mathbb{K}=\left\{a+b \mathbf{i}+c \epsilon+d \mathbf{h}: \mathbf{i}^{2}=-1, \epsilon^{2}=0, \mathbf{h}^{2}=1, a, b, c, d \in \mathbb{R}\right\} .
$$

Let $Z_{1}, Z_{2}$ be any two hybrid numbers, $Z_{1}=a_{1}+b_{1} \mathbf{i}+c_{1} \epsilon+d_{1} \mathbf{h}, Z_{2}=a_{2}+b_{2} \mathbf{i}+c_{2} \epsilon+d_{2} \mathbf{h}$. Then equality, addition, subtraction, multiplication by scaler and the conjugate are defined in the following way.

- $Z_{1}=Z_{2}$ only if $a_{1}=a_{2}, b_{1}=b_{2}, c_{1}=c_{2}, d_{1}=d_{2}$ (equality),

- $Z_{1}+Z_{2}=\left(a_{1}+a_{2}\right)+\left(b_{1}+b_{2}\right) \mathbf{i}+\left(c_{1}+c_{2}\right) \epsilon+\left(d_{1}+d_{2}\right) \mathbf{h}$ (addition), 
- $Z_{1}-Z_{2}=\left(a_{1}-a_{2}\right)+\left(b_{1}-b_{2}\right) \mathbf{i}+\left(c_{1}-c_{2}\right) \epsilon+\left(d_{1}-d_{2}\right) \mathbf{h}$ (subtraction),

- $s Z_{1}=s a_{1}+s b_{1} \mathbf{i}+s c_{1} \epsilon+s d_{1} \mathbf{h}$ (the multiplication by scalar),

- $\overline{Z_{1}}=a_{1}-b_{1} \mathbf{i}-c_{1} \epsilon-d_{1} \mathbf{h}$ (the conjugate of a hybrid number).

The addition operation in the hybrid numbers is both commutative and associative. Zero is the null element. With reference to the addition operation, the inverse element of $Z$ is $-Z=-a-b \mathbf{i}-c \epsilon-d \mathbf{h}$. This shows that $(\mathbb{K} ;+)$ is an Abelian group. The multiplication of hybrid numbers is not commutative, but it has the property of associativity. Table 1 presents products of $1, \mathbf{i}, \epsilon, \mathbf{h}$.

\begin{tabular}{|c||l|l|l|l|}
\hline \hline$\cdot$ & 1 & $\mathbf{i}$ & $\epsilon$ & $\mathbf{h}$ \\
\hline \hline 1 & 1 & $\mathbf{i}$ & $\epsilon$ & $\mathbf{h}$ \\
\hline $\mathbf{i}$ & $i$ & -1 & $1-\mathbf{h}$ & $\epsilon+\mathbf{i}$ \\
\hline$\epsilon$ & $\epsilon$ & $\mathbf{h}+1$ & 0 & $-\epsilon$ \\
\hline $\mathbf{h}$ & $\mathbf{h}$ & $-\epsilon-\mathbf{i}$ & $\epsilon$ & 1 \\
\hline \hline
\end{tabular}

Table 1. The product table for the basis of $\mathbb{K}$

Also, according to the hybridian product, we have $Z \bar{Z}=\bar{Z} Z$. The real number

$$
Z \bar{Z}=\bar{Z} Z=a^{2}+(b-c)^{2}-c^{2}-d^{2}
$$

is called the character of the hybrid number $Z=a+b \mathbf{i}+c \epsilon+d \mathbf{h}$.

For $n \geq 2$, the Fibonacci and Lucas numbers are defined as $F_{n}=F_{n-1}+F_{n-2}, F_{0}=0$, $F_{1}=1$ and $L_{n}=L_{n-1}+L_{n-2}, L_{0}=2, L_{1}=1$. There are interesting identities belongs to the Fibonacci and Lucas numbers in [6]. In recent years, Fibonacci, Lucas, Pell, Jacobsthal and Tribonacci hybrid numbers cover a wide range of interest in modern mathematics as they appear in the comprehensive works of $[1,2,4,5,7,10-16]$. For example, in [4,5] K1zılateş gave the generalizations of the Fibonacci and Lucas hybrid numbers. In [10], Szynal-Liana introduced the Horadam hybrid numbers. In [11,12], the authors also examined the Pell and Pell-Lucas hybrid numbers and the Jacobsthal and Jacobsthal-Lucas hybrid numbers, respectively. In [13], the Fibonacci and Lucas hybrid numbers are defined as

$$
F H_{n}=F_{n}+F_{n+1} \mathbf{i}+F_{n+2} \epsilon+F_{n+3} \mathbf{h}
$$

and

$$
L H_{n}=L_{n}+L_{n+1} \mathbf{i}+L_{n+2} \epsilon+L_{n+3} \mathbf{h} .
$$

Also, the authors found the Binet formulas of these numbers as follows

$$
F H_{n}=\frac{\tilde{\alpha} \alpha^{n}-\tilde{\beta} \beta^{n}}{\alpha-\beta}
$$

and

$$
L H_{n}=\tilde{\alpha} \alpha^{n}+\tilde{\beta} \beta^{n},
$$

where $\alpha=\frac{1+\sqrt{5}}{2}, \beta=\frac{1-\sqrt{5}}{2}, \tilde{\alpha}=1+\alpha \mathbf{i}+\alpha^{2} \epsilon+\alpha^{3} \mathbf{h}, \tilde{\beta}=1+\beta \mathbf{i}+\beta^{2} \epsilon+\beta^{3} \mathbf{h}$. Şentürk et al. [15] gave a lot of properties of the Horadam hybrid numbers and defined the following matrix 


$$
Q_{n}=\left(\begin{array}{cc}
F H_{n+1} & F H_{n} \\
F H_{n} & F H_{n-1}
\end{array}\right)
$$

and obtained the equality $Q_{n}=U^{n} Q_{0}$, where

$$
U=\left(\begin{array}{ll}
1 & 1 \\
1 & 0
\end{array}\right) \text { and } Q_{0}=\left(\begin{array}{cc}
1+\mathbf{i}+2 \epsilon+3 \mathbf{h} & \mathbf{i}+\epsilon+2 \mathbf{h} \\
\mathbf{i}+\epsilon+2 \mathbf{h} & 1+\epsilon+\mathbf{h}
\end{array}\right) .
$$

It is known that

$$
U^{n}=\left(\begin{array}{cc}
F_{n+1} & F_{n} \\
F_{n} & F_{n-1}
\end{array}\right) .
$$

In the light of the $[3,8]$ works, we use the determinant of hybrid matrix similar to the determinant of quaternion matrix whose entries are quaternions. Since the set of all hybrid numbers are not commutative, we give the definitions of the determinant of a hybrid matrix. Let $A$ be a hybrid square matrix. Denote $A$ by

$$
A=\left(\begin{array}{ll}
a_{11} & a_{12} \\
a_{21} & a_{22}
\end{array}\right)
$$

where $a_{i j} \in \mathbb{K}$ for $i=1,2$ and $j=1,2$. The determinant of $A$ is $\operatorname{defined~by~} \operatorname{det} A=a_{11} a_{22}$ $-a_{12} a_{21}$. The above definition is called rule "multiplication from above to down below". Since the set of all hybrid is not commutative, another product direction can be defined. Namely, the definition det $A=a_{22} a_{11}-a_{21} a_{12}$ is called the rule "multiplication from down below to above".

In this study, we obtain the new properties of the Fibonacci and Lucas hybrid numbers. Also, we define the negative subscripts of these numbers. We acquire different Cassini identities for the conjugate of these numbers by two different determinant definitions of a hybrid square matrix.

\section{Main results}

Firstly, the following theorem gives us the relationships between the Fibonacci and Lucas hybrid numbers.

Theorem 2.1. For $n \geq 2$ integers, we have

i) $F H_{n+2}-F H_{n-2}=L H_{n}$,

ii) $5 F H_{n}+L H_{n}=2 L H_{n+1}$.

Proof. i) Since $F_{n+2}-F_{n-2}=L_{n}$, then we get together with equations (1.2) and (1.3)

$$
\begin{aligned}
F H_{n+2}-F H_{n-2} & =F_{n+2}+F_{n+3} \mathbf{i}+F_{n+4} \epsilon+F_{n+5} \mathbf{h}-F_{n-2}-F_{n-1} \mathbf{i}-F_{n} \epsilon-F_{n+1} \mathbf{h} \\
& =L_{n}+L_{n+1} \mathbf{i}+L_{n+2} \epsilon+L_{n+3} \mathbf{h}=L H_{n} .
\end{aligned}
$$

Since the proof of $i i$ ) is same as the proof of $i$ ), we omit these proofs to avoid unnecessary repetition. 
Definition 2.1. The Fibonacci and Lucas hybrid numbers with negative subscripts are defined as

$$
F H_{-n}=F_{-n}+F_{-n+1} \mathbf{i}+F_{-n+2} \epsilon+F_{-n+3} \mathbf{h}
$$

and

$$
L H_{-n}=L_{-n}+L_{-n+1} \mathbf{i}+L_{-n+2} \epsilon+L_{-n+3} \mathbf{h} .
$$

From the equalities $F_{-n}=(-1)^{n+1} F_{n}$ and $L_{-n}=(-1)^{n} L_{n}$, we have

$$
F H_{-n}=\left\{\begin{array}{ll}
-F_{n}+F_{n-1} \mathbf{i}-F_{n-2} \epsilon+F_{n-3} \mathbf{h}, & n \text { is even } \\
F_{n}-F_{n-1} \mathbf{i}+F_{n-2} \epsilon-F_{n-3} \mathbf{h}, & n \text { is odd }
\end{array},\right.
$$

and

$$
L H_{-n}=\left\{\begin{array}{ll}
L_{n}-L_{n-1} \mathbf{i}+L_{n-2} \epsilon-L_{n-3} \mathbf{h}, & n \text { is even } \\
-L_{n}+L_{n-1} \mathbf{i}-L_{n-2} \epsilon+L_{n-3} \mathbf{h}, & n \text { is odd }
\end{array} .\right.
$$

We obtain the binomial summations of the Fibonacci hybrid numbers in the following theorem.

Theorem 2.2. The following identities hold:

i) $\sum_{k=0}^{n}\left(\begin{array}{l}n \\ k\end{array}\right) F H_{k}=F H_{2 n}$,

ii) $\sum_{k=0}^{n}\left(\begin{array}{l}n \\ k\end{array}\right) F H_{k+1}=F H_{2 n+1}$,

iii) $\sum_{k=0}^{n}\left(\begin{array}{l}n \\ k\end{array}\right)(-1)^{k} F H_{k}=(-1)^{n} F H_{-n}$,

iv) $\sum_{k=0}^{n}\left(\begin{array}{l}n \\ k\end{array}\right) F H_{4 k}=3^{n} F H_{2 n}$,

v) $\sum_{k=0}^{n}\left(\begin{array}{l}n \\ k\end{array}\right) 2^{n-k} F H_{5 k}=5^{n} F H_{2 n}$,

vi) $\sum_{k=0}^{n}\left(\begin{array}{l}n \\ k\end{array}\right) 3^{n-k} F H_{6 k}=8^{n} F H_{2 n}$,

vii) $\sum_{k=0}^{n}\left(\begin{array}{l}n \\ k\end{array}\right)(-2)^{k} F H_{2 k}=(-1)^{n} F H_{3 n}$,

viii) $\sum_{k=0}^{n}\left(\begin{array}{l}n \\ k\end{array}\right)(-2)^{k} F H_{5 k}=(-5)^{n} F H_{3 n}$.

Proof. i) From equation (1.4), we write

$$
\begin{aligned}
\sum_{k=0}^{n}\left(\begin{array}{l}
n \\
k
\end{array}\right) F H_{k} & =\sum_{k=0}^{n}\left(\begin{array}{l}
n \\
k
\end{array}\right)\left(\frac{\tilde{\alpha} \alpha^{k}-\tilde{\beta} \beta^{k}}{\alpha-\beta}\right) \\
& =\frac{\tilde{\alpha}}{\alpha-\beta} \sum_{k=0}^{n}\left(\begin{array}{l}
n \\
k
\end{array}\right) \alpha^{k}-\frac{\tilde{\beta}}{\alpha-\beta} \sum_{k=0}^{n}\left(\begin{array}{l}
n \\
k
\end{array}\right) \beta^{k} \\
& =\frac{\tilde{\alpha}}{\alpha-\beta}(1+\alpha)^{n}-\frac{\tilde{\beta}}{\alpha-\beta}(1+\beta)^{n}
\end{aligned}
$$

By considering the well-known equalities $1+\alpha=\alpha^{2}, 1+\beta=\beta^{2}$ and again equation (1.4), we obtain the claimed result.

iii) By considering equations (1.2) and the Binet formula of Fibonacci numbers, we get 


$$
\begin{aligned}
\sum_{k=0}^{n}\left(\begin{array}{l}
n \\
k
\end{array}\right)(-1)^{k} F H_{k}= & \sum_{k=0}^{n}\left(\begin{array}{l}
n \\
k
\end{array}\right)(-1)^{k}\left(F_{k}+F_{k+1} \mathbf{i}+F_{k+2} \epsilon+F_{k+3} \mathbf{h}\right) \\
= & \frac{1}{\alpha-\beta} \sum_{k=0}^{n}\left(\begin{array}{l}
n \\
k
\end{array}\right)\left((-\alpha)^{k}-(-\beta)^{k}\right) \\
& +\frac{\mathbf{i}}{\alpha-\beta} \sum_{k=0}^{n}\left(\begin{array}{l}
n \\
k
\end{array}\right)\left(\alpha(-\alpha)^{k}-\beta(-\beta)^{k}\right) \\
& +\frac{\epsilon}{\alpha-\beta} \sum_{k=0}^{n}\left(\begin{array}{l}
n \\
k
\end{array}\right)\left(\alpha^{2}(-\alpha)^{k}-\beta^{2}(-\beta)^{k}\right) \\
& +\frac{\mathbf{h}}{\alpha-\beta} \sum_{k=0}^{n}\left(\begin{array}{l}
n \\
k
\end{array}\right)\left(\alpha^{3}(-\alpha)^{k}-\beta^{3}(-\beta)^{k}\right) .
\end{aligned}
$$

By taking into account the equalities $\alpha+\beta=1, \alpha \beta=-1$ and Definition 2.1, we obtain

$$
\sum_{k=0}^{n}\left(\begin{array}{l}
n \\
k
\end{array}\right)(-1)^{k} F H_{k}=-F_{n}+F_{n-1} \mathbf{i}-F_{n-2} \epsilon+F_{n-3} \mathbf{h}=(-1)^{n} F H_{-n} .
$$

In the same way, the other parts of the theorem can be proved.

We present the binomial summations of the Lucas hybrid numbers in the following proposition. Since the proof of these summations are similar to the Fibonacci hybrid numbers in the above theorem, we omit the proof.

Proposition 2.1. The equalities are satisfied:

i) $\sum_{k=0}^{n}\left(\begin{array}{l}n \\ k\end{array}\right) L H_{k}=L H_{2 n}$,

ii) $\sum_{k=0}^{n}\left(\begin{array}{l}n \\ k\end{array}\right) L H_{k+1}=L H_{2 n+1}$,

iii) $\sum_{k=0}^{n}\left(\begin{array}{l}n \\ k\end{array}\right)(-1)^{k} L H_{k}=(-1)^{n} L H_{-n}$,

iv) $\sum_{k=0}^{n}\left(\begin{array}{l}n \\ k\end{array}\right) L H_{4 k}=3^{n} L H_{2 n}$,

v) $\sum_{k=0}^{n}\left(\begin{array}{l}n \\ k\end{array}\right) 2^{n-k} L H_{5 k}=5^{n} L H_{2 n}$,

vi) $\sum_{k=0}^{n}\left(\begin{array}{l}n \\ k\end{array}\right) 3^{n-k} L H_{6 k}=8^{n} L H_{2 n}$,

vii) $\sum_{k=0}^{n}\left(\begin{array}{l}n \\ k\end{array}\right)(-2)^{k} L H_{2 k}=(-1)^{n} L H_{3 n}$,

viii) $\sum_{k=0}^{n}\left(\begin{array}{l}n \\ k\end{array}\right)(-2)^{k} L H_{5 k}=(-5)^{n} L H_{3 n}$.

The properties in the following theorem are called convolution product of the Fibonacci and Lucas hybrid numbers.

Theorem 2.3. Let $m \geq 1, n \geq 1$ be integers. Then

i) $F H_{m+1} F H_{n}+F H_{m} F H_{n-1}=F H_{m+n}+F H_{m+n+1} \mathbf{i}+F H_{m+n+2} \epsilon+F H_{m+n+3} \mathbf{h}$,

ii) $L H_{m+1} F H_{n}+L H_{m} F H_{n-1}=L H_{m+n}+L H_{m+n+1} \mathbf{i}+L H_{m+n+2} \epsilon+L H_{m+n+3} \mathbf{h}$. 
Proof. i) Given the matrices $U^{n} Q_{0}, Q_{0} U^{m}$ as equations (1.7) and (1.8), and considering the term $a_{12}$ of the product $\left(Q_{0} U^{m}\right)\left(U^{n} Q_{0}\right)$, which is equal to the term $a_{12}$ of matrix $Q_{0}\left(U^{m+n} Q_{0}\right)$, we get the result.

ii) From the property $\mathrm{FH}_{m+2}-\mathrm{FH}_{m-2}=L H_{m}$ in Theorem 2.1, by considering the term $a_{12}$ of the product $\left(Q_{0}\left(U^{m+2}-U^{m-2}\right)\right)\left(U^{n} Q_{0}\right)$, which is equal to the term $a_{12}$ of matrix $Q_{0}\left(U^{m+n+2} Q_{0}-U^{m+n-2} Q_{0}\right)$, we obtain the result.

If we take $m=n$ in Theorem 2.3, we obtain the following results:

Corollary 2.1. Let $n \geq 1$ be an integer. Then

i) $F H_{n+1} F H_{n}+F H_{n} F H_{n-1}=F H_{2 n}+F H_{2 n+1} \mathbf{i}+F H_{2 n+2} \epsilon+F H_{2 n+3} \mathbf{h}$,

ii) $L H_{n+1} F H_{n}+L H_{n} F H_{n-1}=L H_{2 n}+L H_{2 n+1} \mathbf{i}+L H_{2 n+2} \epsilon+L H_{2 n+3} \mathbf{h}$.

If we take $m=n-1$ in Theorem 2.3, we obtain the following results:

Corollary 2.2. Let $n \geq 2$ be an integer. Then

i) $F H_{n}^{2}+F H_{n-1}^{2}=F H_{2 n-1}+F H_{2 n} \mathbf{i}+F H_{2 n+1} \epsilon+F H_{2 n+2} \mathbf{h}$,

ii) $L H_{n} F H_{n}+L H_{n-1} F H_{n-1}=L H_{2 n-1}+L H_{2 n} \mathbf{i}+L H_{2 n+1} \epsilon+L H_{2 n+2} \mathbf{h}$.

Now, we examine Cassini identities for the conjugates of Fibonacci and Lucas hybrid numbers. For this reason, we present the conjugate of matrix in equation (1.7)

$$
\overline{Q_{0}}=\left(\begin{array}{cc}
1-\mathbf{i}-2 \epsilon-3 \mathbf{h} & -\mathbf{i}-\epsilon-2 \mathbf{h} \\
-\mathbf{i}-\epsilon-2 \mathbf{h} & 1-\epsilon-\mathbf{h}
\end{array}\right) .
$$

Theorem 2.4. Let $n \geq 1$ be an integer. Then

i) $\overline{F H}_{n+1} \overline{F H}_{n-1}-\left(\overline{F H}_{n}\right)^{2}=(-\epsilon-5 \mathbf{h})(-1)^{n}$,

ii) $\overline{F H}_{n-1} \overline{F H}_{n+1}-\left(\overline{F H}_{n}\right)^{2}=(-2 \mathbf{i}-5 \epsilon-3 \mathbf{h})(-1)^{n}$,

iii) $\overline{L H}_{n+1} \overline{L H}_{n-1}-\left(\overline{L H}_{n}\right)^{2}=5(-\epsilon-5 \mathbf{h})(-1)^{n-1}$,

iv) $\overline{L H}_{n-1} \overline{L H}_{n+1}-\left(\overline{L H}_{n}\right)^{2}=5(-2 \mathbf{i}-5 \epsilon-3 \mathbf{h})(-1)^{n-1}$.

Proof. By equation (1.2) and the matrices in (1.8),(2.5), we get

$$
\begin{aligned}
\overline{Q_{0}} U^{n} & =\left(\begin{array}{cc}
1-\mathbf{i}-2 \epsilon-3 \mathbf{h} & -\mathbf{i}-\epsilon-2 \mathbf{h} \\
-\mathbf{i}-\epsilon-2 \mathbf{h} & 1-\epsilon-\mathbf{h}
\end{array}\right)\left(\begin{array}{cc}
F_{n+1} & F_{n} \\
F_{n} & F_{n-1}
\end{array}\right) \\
& =\left(\begin{array}{cc}
\overline{F H}_{n+1} & \overline{F H}_{n} \\
\overline{F H}_{n} & \overline{F H}_{n-1}
\end{array}\right) .
\end{aligned}
$$

i) We acquire the identity after taking the determinant under of the matrices in (2.6) and (2.7) applying the rules "multiplication from above to down below" which mentioned in introduction section. That is,

$$
\overline{F H}_{n+1} \overline{F H}_{n-1}-\left(\overline{F H}_{n}\right)^{2}=(-\epsilon-5 \mathbf{h})(-1)^{n} .
$$


ii) We obtain the identity after taking the determinant under of the matrices in (2.6) and (2.7) applying the rules "multiplication from down below to above" which mentioned in introduction section.

By equations $F_{n+2}-F_{n-2}=L_{n},(1.3)$ and the matrices in (1.8),(2.5), we get

$$
\begin{aligned}
\overline{Q_{0}}\left(U^{n+2}-U^{n-2}\right) & =\left(\begin{array}{cc}
1-\mathbf{i}-2 \epsilon-3 \mathbf{h} & -\mathbf{i}-\epsilon-2 \mathbf{h} \\
-\mathbf{i}-\epsilon-2 \mathbf{h} & 1-\epsilon-\mathbf{h}
\end{array}\right)\left(\begin{array}{cc}
L_{n+1} & L_{n} \\
L_{n} & L_{n-1}
\end{array}\right) \\
& =\left(\begin{array}{cc}
\overline{L H}_{n+1} & \overline{L H}_{n} \\
\overline{L H}_{n} & \overline{L H}_{n-1}
\end{array}\right) .
\end{aligned}
$$

iii) We get the identity after taking the determinant under of the matrices in (2.8) and (2.9) applying the rules "multiplication from above to down below" which were mentioned in the introduction section. That is,

$$
\overline{L H}_{n+1} \overline{L H}_{n-1}-\left(\overline{L H}_{n}\right)^{2}=5(-\epsilon-5 \mathbf{h})(-1)^{n-1} .
$$

$i v$ ) We obtain the identity after taking the determinant under of the matrices in (2.8) and (2.9) applying the rules "multiplication from down below to above" which mentioned in introduction section.

The following theorem gives us the relationships between the Fibonacci hybrid numbers and the conjugates of them.

Theorem 2.5. Let $m \geq 0, n \geq 0$ be integers. Then

i) $F H_{m+n+1}-F H_{m+n+2} \mathbf{i}-F H_{m+n+3} \epsilon-F H_{m+n+4} \mathbf{h}=\overline{F H}_{m+1} F H_{n+1}+\overline{F H}_{m} F H_{n}$,

ii) $\overline{F H}_{m+n+1}+\overline{F H}_{m+n+2} \mathbf{i}+\overline{F H}_{m+n+3} \epsilon+\overline{F H}_{m+n+4} \mathbf{h}=\overline{F H}_{m+1} F H_{n+1}+\overline{F H}_{m} F H_{n}$,

iii) $F H_{m+n+1}-F H_{m+n+2} \mathbf{i}-F H_{m+n+3} \epsilon-F H_{m+n+4} \mathbf{h}=F H_{m+1} \overline{F H}_{n+1}+F H_{m} \overline{F H}_{n}$,

iv) $\overline{F H}_{m+n+1}+\overline{F H}_{m+n+2} \mathbf{i}+\overline{F H}_{m+n+3} \epsilon+\overline{F H}_{m+n+4} \mathbf{h}=F H_{m+1} \overline{F H}_{n+1}+F H_{m} \overline{F H}_{n}$.

Proof. We get the desired identity by equating the pivot elements of the matrices

i) $\overline{Q_{0}}\left(U^{m+n} Q_{0}\right)=\left(\overline{Q_{0}} U^{m}\right)\left(U^{n} Q_{0}\right)$,

ii) $\left(\overline{Q_{0}} U^{m+n}\right) Q_{0}=\left(\overline{Q_{0}} U^{m}\right)\left(U^{n} Q_{0}\right)$,

iii) $\left(Q_{0} U^{m+n}\right) \overline{Q_{0}}=\left(Q_{0} U^{m}\right)\left(U^{n} \overline{Q_{0}}\right)$,

iv) $Q_{0}\left(U^{m+n} \overline{Q_{0}}\right)=\left(Q_{0} U^{m}\right)\left(U^{n} \overline{Q_{0}}\right)$.

If we take $m=n$ in Theorem 2.5, we get the following results by considering equation (1.1):

Corollary 2.3. Let $n \geq 0$ be an integer. Then

i) $\mathrm{FH}_{2 n+1}-\mathrm{FH}_{2 n+2} \mathbf{i}-\mathrm{FH}_{2 n+3} \epsilon-\mathrm{FH}_{2 n+4} \mathbf{h}=2 F_{2 n+1}-L_{2 n+6}$,

ii) $\overline{F H}_{2 n+1}+\overline{F H}_{2 n+2} \mathbf{i}+\overline{F H}_{2 n+3} \epsilon+\overline{F H}_{2 n+4} \mathbf{h}=2 F_{2 n+1}-L_{2 n+6}$.

The following proposition gives us the relationships between the Lucas hybrid numbers and the conjugates of them. The proof similar to the proofs of the Theorem 2.4 and Theorem 2.5. 
Proposition 2.2. Let $m \geq 0, n \geq 0$ be integers. Then

i) $L H_{m+n+1}-L H_{m+n+2} \mathbf{i}-L H_{m+n+3} \epsilon-L H_{m+n+4} \mathbf{h}=\overline{L H}_{m+1} F H_{n+1}+\overline{L H}_{m} F H_{n}$,

ii) $\overline{L H}_{m+n+1}+\overline{L H}_{m+n+2} \mathbf{i}+\overline{L H}_{m+n+3} \epsilon+\overline{L H}_{m+n+4} \mathbf{h}=\overline{L H}_{m+1} F H_{n+1}+\overline{L H}_{m} F H_{n}$,

iii) $L H_{m+n+1}-L H_{m+n+2} \mathbf{i}-L H_{m+n+3} \epsilon-L H_{m+n+4} \mathbf{h}=L H_{m+1} \overline{F H}_{n+1}+L H_{m} \overline{F H}_{n}$,

iv) $\overline{L H}_{m+n+1}+\overline{L H}_{m+n+2} \mathbf{i}+\overline{L H}_{m+n+3} \epsilon+\overline{L H}_{m+n+4} \mathbf{h}=L H_{m+1} \overline{F H}_{n+1}+L H_{m} \overline{F H}_{n}$.

If we take $m=n$ in Proposition 2.2, we obtain the following results by considering equation (1.1):

Corollary 2.4. Let $n \geq 0$ be an integer. Then

i) $L H_{2 n+1}-L H_{2 n+2} \mathbf{i}-L H_{2 n+3} \epsilon-L H_{2 n+4} \mathbf{h}=-L_{2 n}-2 L_{2 n+6}$,

ii) $\overline{L H}_{2 n+1}+\overline{L H}_{2 n+2} \mathbf{i}+\overline{L H}_{2 n+3} \epsilon+\overline{L H}_{2 n+4} \mathbf{h}=-L_{2 n}-2 L_{2 n+6}$.

\section{Conclusion}

In this paper, the Fibonacci and Lucas hybrid numbers have been investigated. Many of the properties of these numbers are proved by the fundamental algebraic operations and simple matrix algebra. Actually, the results presented here have the potential to motivate further studies of the subject of the Horadam hybrinomials including Fibonacci and Lucas hybrid numbers.

\section{Acknowledgements}

The author would like to thank the anonymous reviewers for their useful comments and suggestions.

\section{References}

[1] Catarino, P. (2019). On k-Pell hybrid numbers. Journal of Discrete Mathematical Sciences and Cryptography, 22(1), 83-89.

[2] Cerda-Morales, G. (2018). Investigation of Generalized Hybrid Fibonacci Numbers and Their Properties. arXiv preprint, arXiv:1806.02231.

[3] Irmak, N. (2020). More identities for Fibonacci and Lucas quaternions. Communications Faculty of Sciences University of Ankara Series Al Mathematics and Statistics, 69(1), 369-375.

[4] Kızılateş, C. (2020). A new generalization of Fibonacci hybrid and Lucas hybrid numbers. Chaos, Solitons and Fractals, 130, 109449. 
[5] Kızılateş, C. (2020). A Note on Horadam Hybrinomials. Preprint, doi:10.20944/ preprints202001.0116.v1.

[6] Koshy, T. (2001). Fibonacci and Lucas Numbers with Applications. John Wiley and Sons Inc., NY.

[7] Liana, M., Szynal-Liana, A., \& Włoch, I. (2019). On Pell Hybrinomials. Miskolc Mathematical Notes, 20(2), 1051-1062.

[8] Morais, J. P., Georgiev, S., \& Spröbig, W. (2014). Real Quaternionic Calculus Handbook. Birkhauser, London.

[9] Özdemir, M. (2018). Introduction to Hybrid Numbers. Advances in Applied Clifford Algebras, 28(11), 1-32.

[10] Szynal-Liana, A. (2018). The Horadam Hybrid Numbers. Discussiones Mathematicae General Algebra and Applications, 38(1), 91-98.

[11] Szynal-Liana, A., \& Włoch, I. (2018). On Pell and Pell-Lucas Hybrid Numbers. Commentationes Mathematicae, 58(1-2), 11-17.

[12] Szynal-Liana, A., \& Włoch, I. (2019). On Jacobsthal and Jacobsthal-Lucas Hybrid Numbers. Annales Mathematicae Silesianae, 33(1), 276-283.

[13] Szynal-Liana, A., \& Włoch, I. (2019). The Fibonacci hybrid numbers. Utilitas Mathematica, $110,3-10$.

[14] Szynal-Liana, A., \& Włoch, I. (2020). Introduction to Fibonacci and Lucas hybrinomials. Complex Variables and Elliptic Equations, 65(10), 1736-1747.

[15] Şentürk, T.D., Bilgici, G., Daşdemir, A., \& Ünal, Z. (2020). A Study on Horadam Hybrid Numbers. Turkish Journal of Mathematics, 44(4), 1212-1221.

[16] Taşyurdu, Y. (2019). Tribonacci and Tribonacci-Lucas Hybrid Numbers. International Journal of Contemporary Mathematical Sciences, 14(4), 245-254. 